\title{
Aging Decreases the Capacity of Human Skin to Produce Vitamin $D_{3}$
}

\author{
Julia MacLaughlin and Michael F. Holick \\ Vitamin D Laboratory, Endocrine Unit, Massachusetts General Hospital and Harvard Medical School, Boston, Massachusetts 02114; and \\ Vitamin D and Bone Metabolism Laboratory, USDA/Human Nutrition Research Center on Aging, Tufts University, Boston,
}

Massachusetts 02111

\begin{abstract}
An evaluation of surgically obtained skin (age range, 8-92 yr) revealed that there is an age-dependent decrease in the epidermal concentrations of provitamin $\mathbf{D}_{\mathbf{3}}$ (7-dehydrocholesterol). To ascertain that aging indeed decreased the capacity of human skin to produce vitamin $D_{3}$, some of the skin samples were exposed to ultraviolet radiation and the content of previtamin $D_{3}$ was determined in the epidermis and dermis. The epidermis in the young and older subjects was the major site for the formation of previtamin $D_{3}$, accounting for $>\mathbf{8 0} \%$ of the total previtamin $D_{3}$ that was produced in the skin. A comparison of the amount of previtamin $D_{3}$ produced in the skin from the 8- and 18-yr-old subjects with the amount produced in the skin from the 77- and 82-yr-old subjects revealed that aging can decrease by greater than twofold the capacity of the skin to produce previtamin $D_{3}$. Recognition of this difference may be extremely important for the elderly, who infrequently expose a small area of skin to sunlight and who depend on this exposure for their vitamin D nutritional needs.
\end{abstract}

\section{Introduction}

It is well established that aging is accompanied by a decrease in bone mass (1). Although osteopenia in the elderly is mainly due to osteoporosis, a significant number of patients suffering from hip fractures have occult osteomalacia (1-5). In the United Kingdom, several investigators have demonstrated, by histologic evaluation of bone biopsies, that $20-30 \%$ of women and up to $40 \%$ of men with fractures of the proximal aspect of the femur have occult osteomalacia (3-5). Similarly, in the United States, Sokoloff reported low-grade osteomalacia in $25 \%$ of 32 elderly, normocalcemic patients with intracapsular fractures (6). Recently, Doppelt et al. (7) reported that an evaluation of 172 patients admitted to the hospital with hip fractures revealed that up to $40 \%$ had low or undetectable concentrations of 25 -hydroxyvitamin D (25-OH-D), and, of the bone biopsies obtained, $30 \%$ had wide osteoid seams suggestive of osteomalacia. Vitamin $\mathrm{D}$ deficiency in the elderly is caused by many factors including (a) deprivation of sunlight, (b) avoidance of dairy products, owing either to low income or intolerance due to lactase deficiency, and $(c)$ intestinal malabsorption of fat-soluble vitamin $D$. In the United Kingdom, where vitamin D supplementation is not widely practiced, the body stores of vitamin $\mathrm{D}$ are more depen-

Address correspondence and reprint requests to Dr. Holick, USDA/Human Nutrition Center on Aging at Tufts University, Boston, MA 02111.

Received for publication 5 November 1984 and in revised form 28 June 1985.

J. Clin. Invest.

(c) The American Society for Clinical Investigation, Inc.

$0021-9738 / 85 / 10 / 1536 / 03 \$ \$ 1.00$

Volume 76, October 1985, 1536-1538 dent on the cutaneous synthesis of vitamin $D_{3}$. There is a fall in plasma 25-OH-D concentrations with age in Britain (1), which is considered attributable to decreased exposure to sunlight rather than decreased intake of vitamin $D$.

In adults, the epidermis is the major site for sunlight-mediated vitamin $\mathrm{D}_{3}$ synthesis. During exposure to sunlight, ultraviolet photons (with energies between 290 and $315 \mathrm{~nm}$ ) penetrate into the epidermis and photolyze 7-dehydrocholesterol to previtamin $D_{3}(8)$. Once formed, previtamin $D_{3}$ slowly equilibrates to vitamin $D_{3}$, which, in turn, is translocated into the circulation (8).

It is well known that there are numerous dermatologic changes that occur with aging, including the clinical observation that many osteoporotic women have thin and frail skin. To determine whether aging affected the capacity of human skin to synthesize vitamin $\mathrm{D}_{3}$, we determined $(a)$ the concentration of 7-dehydrocholesterol (provitamin $\mathrm{D}_{3}$ ) in a defined area of the epidermis and dermis of individuals of varying age, and $(b)$ the formation of previtamin $D_{3}$ in the skin after exposure to ultraviolet radiation.

\section{Methods}

Caucasian human skin samples (ages 8-92) skin type III were obtained at random at the time of surgery usually from patients undergoing reduction mammoplasty or limb amputation. The subcutaneous fat was removed and the skin was cut into $6.2-\mathrm{cm}^{2}$ pieces. The skin samples were either irradiated with $295 \pm 5 \mathrm{~nm}$ radiation $\left(0.15 \mathrm{~J} / \mathrm{cm}^{2}\right)$ or kept in the dark as controls as previously described (8). The epidermis was either scraped mechanically from the dermis or separated into the basal-cell layer and remaining epidermis (8). The lipids were extracted from these tissues with $8 \%$ ethylacetate/hexane and analyzed for 7-dehydrocholesterol and its photoproducts by high performance liquid chromatography as previously described (8).

\section{Results}

Table I and Fig. 1 demonstrate the effect of aging on the wet weight of the whole skin and epidermis and on the 7-dehydrocholesterol concentrations in the whole epidermis and dermis. The epidermal concentrations of 7-dehydrocholesterol show an age-dependent decrease that is not accounted for by an agerelated decrease in the total mass of the epidermis (Table I), whereas the dermal concentrations of 7-dehydrocholesterol remain relatively constant in the 28 subjects who ranged in age from 8 to 92 yr. To determine whether the age-dependent decrease of 7-dehydrocholesterol in the epidermis was due to a decrease in the concentration of 7-dehydrocholesterol in the actively reproducing layer of the epidermis (which was previously shown to be responsible for the source of provitamin $D_{3}$ in the epidermis [8]), the concentration of 7-dehydrocholesterol in the stratum basale was determined in some of the skin samples. As shown in Fig. 1, there is an age-dependent decrease in the 7dehydrocholesterol in the stratum basale that parallels the decrease in the entire epidermis. 
Table I. Effect of Aging on the Weight of the Whole Skin and Epidermis and on the Concentration of 7-Dehydrocholesterol (7-DHC) in the Epidermis *

\begin{tabular}{lllll}
\hline Age & $\begin{array}{l}\text { Wet weight of } \\
\text { whole skin }\end{array}$ & $\begin{array}{l}\text { Wet weight of } \\
\text { epidermis }\end{array}$ & 7-DHC & 7-DHC \\
\hline$y r$ & $\mathrm{~g} / \mathrm{cm}^{2}$ & $\mathrm{~g} / \mathrm{cm}^{2}$ & $\mathrm{ng} / \mathrm{cm}^{2}$ & $\mu \mathrm{g} / \mathrm{g}$ \\
21 & 0.77 & 0.15 & 1747 & 11.65 \\
29 & 0.64 & 0.14 & 1614 & 11.53 \\
47 & 0.66 & 0.15 & 1350 & 9.00 \\
58 & 0.72 & 0.16 & 1409 & 8.81 \\
77 & 0.58 & 0.14 & 338 & 2.41 \\
88 & 0.58 & 0.12 & 838 & 6.98 \\
\hline
\end{tabular}

* Surgically obtained human skin was weighed and separated and the 7-DHC concentrations quantitated as previously described (8).

To ascertain that aging indeed decreased the capacity of human epidermis to produce previtamin $D_{3}$, we determined the previtamin $D_{3}$ concentrations in the epidermis and dermis after exposing representative skin samples to ultraviolet radiation (Table II). The epidermis in young and older subjects was the major site for previtamin $D_{3}$ synthesis in the skin, accounting for $>80 \%$ of the previtamin $D_{3}$ that was produced in the skin. Less than $20 \%$ of previtamin $D_{3}$ synthesis occurred in the dermis in both age groups. There was a dramatic decline in the ability of the epidermis from the subjects 77- and 82-yr-old to produce previtamin $D_{3}$. A comparison of the total amount of previtamin $D_{3}$ produced in the epidermis and dermis from the 8- and 18yr-olds with the amount produced in the epidermis and dermis of the 77- and 82-yr-old subjects revealed that aging can decrease by greater than twofold the capacity of the skin to produce previtamin $\mathrm{D}_{3}$ (Table II).

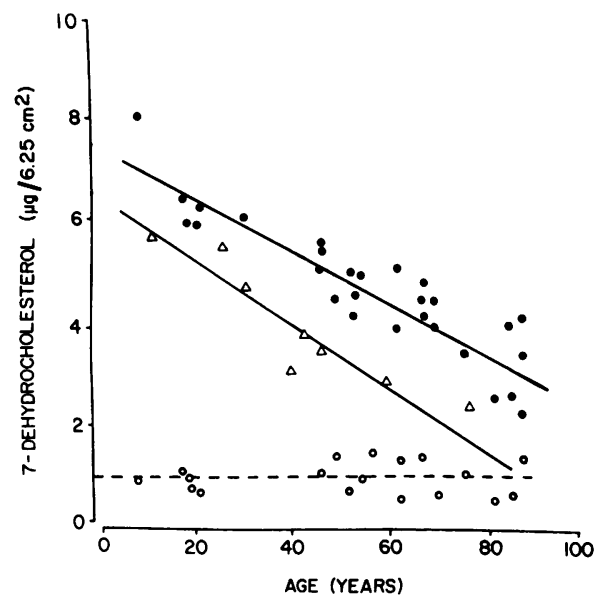

Figure 1. Effect of aging on 7-dehydrocholesterol concentrations in human epidermis and dermis. Concentrations of 7-dehydrocholesterol (provitamin $\mathrm{D}_{3}$ ) per unit area of human epidermis $(\bullet)$, stratum basale $(\Delta)$, and dermis $(0)$ obtained from surgical specimens from donors of various ages. A linear regression analysis revealed slopes of -0.05 , -0.06 , and -0.0005 for the epidermis $(r=-0.89)$, stratum basale $(r$ $=-0.92)$, and dermis $(r=-0.04)$, respectively. The slopes of the epidermis and stratum basale are significantly different from the slope of the dermis $(P<0.001)$.
Table II. 7-Dehydrocholesterol (7-DHC) Content Before

Exposure to Ultraviolet Radiation and Previtamin

$D_{3}$ (pre $D_{3}$ ) Content After Exposure to Ultraviolet

Radiation * and the Percentage of Pre $\mathrm{D}_{3}$ Formed in the Epidermis and Dermis Relative to the 8-yr-old Subject

\begin{tabular}{|c|c|c|c|c|c|c|}
\hline \multirow[b]{3}{*}{ Age } & \multirow{2}{*}{\multicolumn{2}{|c|}{ Epidermis }} & \multirow{2}{*}{\multicolumn{2}{|c|}{ Dermis }} & \multicolumn{2}{|c|}{ Epidermis and dermis } \\
\hline & & & & & \multirow[b]{2}{*}{ preD $_{3}$} & \multirow{2}{*}{$\begin{array}{l}\text { \% Formation } \\
\text { pre } \mathrm{D}_{3} \text { compare } \\
\text { with 8-yr-old }\end{array}$} \\
\hline & 7-DHC & preD $_{3}$ & 7-DHC & preD $_{3}$ & & \\
\hline & $\mathrm{ng} / \mathrm{cm}^{2}$ & $\mathrm{ng} / \mathrm{cm}^{2}$ & $\mathrm{ng} / \mathrm{cm}^{2}$ & $\mathrm{ng} / \mathrm{cm}^{2}$ & $\mathrm{ng} / \mathrm{cm}^{2}$ & \\
\hline 8 & 1,308 & 406 & 1,800 & 36 & 442 & 100 \\
\hline 18 & 1,056 & 346 & 1,125 & 22 & 368 & 80 \\
\hline 77 & 605 & 144 & 1,630 & 24 & 168 & 37 \\
\hline 77 & 490 & 141 & - & - & - & - \\
\hline 82 & 659 & 163 & 1,040 & 20 & 183 & 40 \\
\hline
\end{tabular}

* In $1 \mathrm{~cm}^{2}$ of human epidermis and dermis.

\section{Discussion}

There is mounting evidence that the elderly persons in the United States, Israel, and Europe have an increased risk of developing vitamin D deficiency $(1-7,9-12)$. One of the possible causes for this increase is that aging decreases the capacity of the skin to produce vitamin $D_{3}$. Lester et al. (13) were among the first to imply that aging decreased the efficiency of vitamin $\mathrm{D}_{3}$ formation in the skin; they observed that the circulating concentrations of 25-OH-D are lower in nonhousebound elderly, even during the summer months, when compared with healthy young British subjects. Corless et al. (14) were the first to examine the response of plasma 25-OH-D to different exposures of ultraviolet radiation in geriatric patients. They found that multiple exposures to suberythemal doses of ultraviolet radiation could bring the plasma 25-OH-D into the normal range. Because they did not have younger subjects in their study for comparison, they warned that care should be taken in applying their results to a younger population, for there was some evidence that 25-hydroxylation of vitamin D declines with age. Davie and Lawson (15) found that plasma 25-OH-D concentrations increased to comparable levels in a group of young and old subjects who received multiple exposures of ultraviolet radiation either over an area 600-900 $\mathrm{cm}^{2}$ on their backs or over their entire bodies. Despite these findings, it remains a fact that the elderly are more at risk for developing vitamin $\mathrm{D}$ deficiency and osteomalacia $(1-7,9-12$, $14,15)$. It was suggested that this is a result of the failure of aged individuals to go outdoors or from the possibility that the skin of an aged individual requires a greater dose of ultraviolet radiation to synthesize vitamin $D_{3}(15)$.

Because vitamin $D$ is absolutely essential for the development and maintenance of a healthy skeletal structure in humans, those who do not obtain adequate vitamin $\mathrm{D}$ nutrition from their diet must obtain this essential hormone from exposure of the skin to environmental sunlight. Thus, it is important to know whether aging in any way influences the capacity of the skin to produce vitamin $D_{3}$. It is well established that, after about the age of 20 , skin thickness decreases linearly with age (16). The age-related changes in the skin principally occur in the dermis. The entire elastic fiber structure in the papillary dermis shrinks and sags, and the number of superficial capillary loops and tufts in the papillary body just beneath the epidermis is markedly reduced (17).

To determine whether aging affected the synthetic capacity 
of human skin to produce vitamin $D_{3}$, we determined the amount of its precursor, 7-dehydrocholesterol, in a defined area of epidermis and dermis. As shown in Fig. 1, there is a dramatic age-related decline in the 7-dehydrocholesterol content in the epidermis, whereas the concentrations of the vitamin $D_{3}$ precursor in the dermis do not appreciably change. This marked decline, however, could not be accounted for by the effect that aging has on the total mass of the epidermis (Table I). Furthermore, to be certain that the significant decrease in the content of epidermal 7-dehydrocholesterol would lead to a decrease in the production of vitamin $D_{3}$, a few representative samples of skin from the young and old subjects were exposed to ultraviolet radiation, and the amount of previtamin $\mathrm{D}_{3}$ was determined in the epidermis and dermis. As can be seen in Table II, the total amount of previtamin $D_{3}$ that was produced in the epidermis and dermis of the young subjects was at least two times greater than when compared with that of the elderly subjects.

Hence, although the elderly are able to photosynthesize previtamin $D_{3}$, their capacity to do so is markedly diminished when compared with that of a young person. Therefore, if the elderly frequently expose a significant area of skin (such as sunbathing in a swimming suit) to sunlight, they probably can maintain adequate vitamin $\mathrm{D}$ nutriture without dietary vitamin $D_{3}$, as suggested by the studies of Davie and Lawson (15). For those elderly, however, who do not obtain adequate vitamin D nutrition from their diet and who infrequently expose a very small area of skin (such as hands and face) to sunlight, the greater than twofold decline in their epidermal stores of 7-dehydrocholesterol may very well put them at risk for development of vitamin D deficiency and osteomalacia. These individuals would benefit by increasing their dietary intake of vitamin $\mathrm{D}$ and by increasing their exposure to the sun.

\section{Acknowledgments}

We are indebted to Dr. A. L. Schiller and Dr. R. M. Goldwyn for some of the skin samples.

This work was supported in part by National Institutes of Health grants AM27334, AG02918, and AG04390.

\section{References}

1. Nordin, B. E. C., M. Peacock, J. Aaron, R. G. Crilly, P. J. Heyburn, A. Horsman, and D. Marshall. 1980. Osteoporosis and osteomalacia. Clin. Endocrinol. Metab. 9:177.
2. Exton-Smith, A. N., H. M. Hodkinson, and B. R. Stanton. 1966. Nutrition and metabolic bone disease in old age. Lancet. II:999-1001.

3. Chalmers, J., D. H. Conacher, D. L. Gardner, and P. J. Scott. 1967. Osteomalacia-a common disease in elderly women. J. Bone Jt. Surg. 49B:403-423.

4. Jenkins, D. H. R., J. G. Roberts, D. Webster, and E. O. Williams. 1973. Osteomalacia in elderly patients with fracture of the femoral neck. J. Bone Jt. Surg. 55B:575-580.

5. Aaron, J. E., J. C. Gallagher, J. Anderson, L. Stasiak, E. B. Longton, B. E. C. Nordin, and M. Nicholson. 1974. Frequency of osteomalacia and osteoporosis in fractures of the proximal femur. Lancet. I:229-231.

6. Sokoloff, L. 1978. Occult osteomalacia in American (USA) patients with fracture of the hip. Am. J. Surg. Pathol. 2:21-30.

7. Doppelt, S. H., R. M. Neer, M. Daly, L. Bourret, A. Schiller, and M. F. Holick. 1983. Vitamin D deficiency and osteomalacia in patients with hip fractures. Orthop. Trans. 7:512-513.

8. Holick, M. F., J. A. MacLaughlin, M. B. Clark, S. A. Holick, J. T. Potts, Jr., R. R. Anderson, I. H. Blank, J. A. Parrish, and P. Elias. 1980. Photosynthesis of previtamin $D_{3}$ in human skin and the physiologic consequences. Science (Wash. DC). 210:203-205.

9. Omdahl, J. L., P. J. Garry, L. A. Hunsaker, W. C. Hunt, and J. S. Goodwin. 1982. Nutritional status in a healthy elderly population: vitamin D. Am. J. Clin. Nutr. 36:1225-1233.

10. Weisman, Y., R. J. Schen, Z. Eisenberg, S. Edelstein, and A. Harell. 1981. Inadequate status and impaired metabolism of vitamin D in the elderly. Isr. J. Med. Sci. 17:19-21.

11. Datani, J. T., A. N. Exton-Smith, and J. M. L. Stephen. 1984. Vitamin D status of the elderly in relation to age and exposure to sunlight. Hum. Nutrit.:Clin. Nutrit. 38C:131-137.

12. Somerville, P. J., W. K. Lien, and M. Kaye. 1977. The calcium and vitamin $\mathrm{D}$ status in an elderly female population and their response to administered supplemental vitamin $\mathrm{D}_{3}$. J. Gerontol. 32:659-662.

13. Lester, E., R. K. Skinner, and M. R. Mills. 1977. Seasonal variation in serum-25-hydroxyvitamin-D in the elderly in Britain. Lancet. I:979980.

14. Corless, D., S. P. Gupta, S. Switala, B. J. Boucher, J. M. Barragry, and R. D. Cohen. 1978. Response of plasma-25-hydroxyvitamin D to ultraviolet irradiation in long-stay geriatric patients. Lancet. I:649-651.

15. Davie, M., and D. E. M. Lawson. 1980. Assessment of plasma 25-hydroxyvitamin $\mathrm{D}$ response to ultraviolet irradiation over a controlled area in young and elderly subjects. Clin. Sci. 58:235-242.

16. Tan, C. Y., B. Statham, R. Marks, and P. A. Payne. 1982. Skin thickness measurement by pulsed ultrasound: its reproducibility, validation, and variability. Br. J. Dermatol. 106:657-667.

17. Montagna, W., and K. Carlisle. 1979. Structural changes in aging human skin. J. Invest. Dermatol. 73:47-53. 